\title{
Observational study of people infected with SARS-Cov-2, treated with amantadine
}

\author{
Gonzalo Emiliano Aranda-Abreu ${ }^{1}$ (1) - José D. Aranda-Martínez ${ }^{2} \cdot$ Ramiro Araújo $^{3} \cdot$ María Elena Hernández-Aguilar $^{1}$. \\ Deissy Herrera-Covarrubias ${ }^{1} \cdot$ Fausto Rojas-Durán ${ }^{1}$
}

Received: 15 August 2020 / Revised: 16 September 2020 / Accepted: 1 October 2020 / Published online: 10 October 2020

(c) Maj Institute of Pharmacology Polish Academy of Sciences 2020

\begin{abstract}
Background We conducted an observational study of 15 patients from a Southeastern area of Mexico with symptoms compatible with SARS-Cov-2, which were treated with the antiviral amantadine.

Methodology In this study, data were collected from 15 individuals with clinical symptoms of COVID-19 infection, which were treated on an ambulatory basis with $100 \mathrm{mg}$ of amantadine for a period of 14 days.

Results This drug demonstrated its effectiveness, as patients recovered successfully with this treatment without the necessity of attending a hospital to use mechanical ventilation. All patients developed IgG antibodies to SARS-Cov-2.

Conclusion Amantadine can be used as a viable and cost-effective alternative for treating people with severe acute respiratory syndrome (SARS-Cov-2) on an ambulatory basis, while the vaccine is not available.
\end{abstract}

Keywords Amantadine $\cdot$ SARS-cov- $2 \cdot$ Celecoxib $\cdot$ Azithromycin

\section{Introduction}

The SARS-Cov-2 pandemic has left more than 40,000 deaths in Mexico [1]; this is probably because there is no effective treatment that can stop the coronavirus worldwide. Studies have been conducted with hydroxychloroquine in combination with azithromycin; however, this has not been

Gonzalo Emiliano Aranda-Abreu

garanda@uv.mx

José D. Aranda-Martínez

g_aranda@hotmail.com

Ramiro Araújo

ramirogeraldesaraujo@ hotmail.com

María Elena Hernández-Aguilar

elenahernandez@uv.mx

Deissy Herrera-Covarrubias

dherrera@uv.mx

Fausto Rojas-Durán

frojas@uv.mx

1 Universidad Veracruzana/Centro de Investigaciones Cerebrales, Xalapa, Veracruz, Mexico

2 Centro Médico Cordobés, Córdoba, Veracruz, México

3 Hospital Da Lapa, Porto, Portugal successful [2]. Studies have also been done with the antiparasitic Ivermectin, but the results are still controversial [3].

The use of antivirals has been approved by the FDA, such as Remdesivir [4], which is an adenosine nucleotide analogue that disrupts viral replication and inhibits the RNA polymerase enzyme. However, its use requires hospital assistance, since it is administered intravenously, which increases treatment costs due to hospital attention.

In 1973 amantadine was approved by the FDA for the treatment of motor symptoms in patients with Parkinson's disease [5]. In 1976, amantadine was developed for the treatment of influenza H1N1 [6, 7]; however, this drug is not recommended for the treatment of influenza, because it mutates and loses sensitivity to the drug [8]. Amantadine has been found to have several benefits, such as being used as an antiparkinsonian, as it is involved in the process of dopamine release [9]. It is important to note that patients with Parkinson's disease, who are being treated with amantadine, have not shown the symptomatology of COVID-19 infection [10, 11]. It is important to mention that the neurological indications of amantadine are not related to its antiviral activity.

Molecular docking studies have shown that amantadine could enter the SARS-Cov-2 E-channel and interfere with the release of its viral content into the cell, thus inhibiting its propagation [12]. Other studies have shown that amantadine 
as a treatment for COVID-19 infection has been effective, and the patients have recovered successfully, without the necessity to go to a hospital [13].

In this observational study, 15 patients were treated in an ambulatory setting in Southern Mexico with amantadine, and it was shown to be effective for the successful recovery of SARS-Cov-2 infected patients.

\section{Patients and methodology}

\section{Study population and data collection}

This study included a sample of 15 patients of different ages, who presented a symptomatology compatible with SARSCov-2. No serological studies were available at the time of the study. Data were collected between April 1 and July 25, 2020, using a questionnaire about the symptoms presented, as well as their chronic diseases. IgM and IgG antibodies were detected with a labclinics ELISA kit (Barcelona, Spain). The report followed the procedures of the observational studies in epidemiology (STROBE) [14].

The inclusion criteria were as follows.

a. Patient consent to participate in the study.

b. Desire to participate in the study.

c. Male or female over 18 years of age.

d. Clinical symptoms compatible with SARS-Cov-2

Exclusion criteria.

a. Legal incapacity.

b. Medical or psychological condition in which the investigator's opinion would not allow completion of the questionnaire.

c. Invasive ventilation.

\section{Case studies}

Ambulatory patients who stayed at home during the quarantine period in a small town in Southeastern Mexico in the state of Tabasco, Mexico.

\section{Objectives of the study}

The main objective of this observational study was to determine if treatment with the drug amantadine would be effective for ambulatory treatment of people manifesting symptoms of COVID-19 infection.

Family members who lived with the patients were suggested to take amantadine prophylactically, $100 \mathrm{mg}$ once a day.
Table 1 Characteristics of the patients and their major comorbidities

\begin{tabular}{ll}
\hline Variable & Patients $n=15$ \\
\hline Age (years), mean \pm SD & $48.2 \pm 16.62(20-82)$ \\
Gender, $n(\%)$ & \\
Male & $5(33.3)$ \\
Female & $10(66.6)$ \\
Major comorbities $n(\%)$ & \\
Obesity & $1(6.6)$ \\
Hypertension & $4(26.6)$ \\
Cardiac disease & $1(6.6)$ \\
Diabetes mellitus & $3(20)$ \\
Renal disease & $0(0)$ \\
Asthma & $0(0)$ \\
Pulmonary disease & $0(0)$ \\
\hline
\end{tabular}

Table 2 Symptomatology presented by the patients

\begin{tabular}{lr}
\hline Symptom, $n(\%)$ & Total \\
\hline Olfactory dysfunction & $10(66.6)$ \\
Gustatory dysfunction & $10(66.6)$ \\
Fever & $14(93.3)$ \\
Dry cough & $14(93.3)$ \\
Dyspnea & $14(93.3)$ \\
Headache & $14(93.3)$ \\
Diarrhea & $5(33.3)$ \\
Nausea & $14(93.3)$ \\
Nasal congestion & $14(93.3)$ \\
\hline
\end{tabular}

\section{Results}

\section{Demographic and clinical characteristics}

A total of 15 patients over 18 years of age with clinical symptoms compatible with SARS-Cov- 2 were included in this study. Table 1 shows the characteristics of the patients and their major comorbidities. The average age of the people in the study was 48 years \pm 16 . Five of them were men and 10 were women. Four per cent suffered from hypertension and three per cent from diabetes mellitus. Only one person was reported to have a heart disease.

Table 2 shows the symptomatology presented by the patients when they were infected with the coronavirus. Most of them presented fever, dyspnea, a headache, nausea, nasal congestion. Ten of them lost their sense of smell and taste.

Table 3 shows the treatment they were recommended to follow. The 15 patients received $100 \mathrm{mg}$ of amantadine, one tablet in the morning and one in the afternoon, over a period of 14 days. Most of them received $500 \mathrm{mg}$ 
Table 3 Treatment protocol for patients

\begin{tabular}{|c|c|c|c|c|c|c|c|c|c|c|c|c|c|c|c|}
\hline & 1 & 2 & 3 & 4 & 5 & 6 & 7 & 8 & 9 & 10 & 11 & 12 & 13 & 14 & 15 \\
\hline Amantadine $100 \mathrm{mg}$ twice a day for 14 days & $\checkmark$ & $\checkmark$ & $\checkmark$ & $\checkmark$ & $\checkmark$ & $\checkmark$ & $\checkmark$ & $\checkmark$ & $\checkmark$ & $\checkmark$ & $\checkmark$ & $\checkmark$ & $\checkmark$ & $\checkmark$ & $\checkmark$ \\
\hline $\begin{array}{l}\text { Azithromycin }(500 \mathrm{mg}) \\
\text { Daily for } 6 \text { days }\end{array}$ & $\checkmark$ & $x$ & $\checkmark$ & $\checkmark$ & $\checkmark$ & $\checkmark$ & $\checkmark$ & $\checkmark$ & $\checkmark$ & $\checkmark$ & $\checkmark$ & $\checkmark$ & $\checkmark$ & $\checkmark$ & $\checkmark$ \\
\hline Celecoxib (200 mg) Daily for 6 days & $\checkmark$ & $x$ & $\checkmark$ & $\checkmark$ & $\checkmark$ & $\checkmark$ & $\checkmark$ & $\checkmark$ & $\checkmark$ & $\checkmark$ & $\checkmark$ & $\checkmark$ & $\checkmark$ & $\checkmark$ & $\checkmark$ \\
\hline $\begin{array}{l}\text { Aspirin }(500 \mathrm{mg}) \\
\text { Daily for } 6 \text { days }\end{array}$ & $\checkmark$ & $x$ & $\checkmark$ & $\checkmark$ & $\checkmark$ & $\checkmark$ & $\checkmark$ & $\checkmark$ & $\checkmark$ & $\checkmark$ & $\checkmark$ & $\checkmark$ & $\checkmark$ & $\checkmark$ & $\checkmark$ \\
\hline $\begin{array}{l}\text { Ipratropium bromide, salbutamol }(0.5 \mathrm{mg} \text {, } \\
2.5 \mathrm{mg} / 2.5 \mathrm{~mL}) \text { to nebulize } \\
\text { 3-Nebulizaciones daily for } 5 \text { days }\end{array}$ & $\checkmark$ & $x$ & $x$ & $\checkmark$ & $x$ & $x$ & $x$ & $x$ & $x$ & $\checkmark$ & $x$ & $x$ & $x$ & $x$ & $x$ \\
\hline Oxygen mask 4 Lpm & $\checkmark$ & $x$ & $x$ & $x$ & $x$ & $x$ & $x$ & $x$ & $x$ & $\checkmark$ & $x$ & $x$ & $x$ & $x$ & $x$ \\
\hline IgG SARS-Cov-2 & + & + & + & + & + & + & + & + & + & + & + & + & + & + & + \\
\hline IgM SARS-Cov-2 & - & - & - & - & - & - & - & - & - & - & - & - & - & - & - \\
\hline
\end{tabular}

of azithromycin for a period of 6 days. They received celecoxib $200 \mathrm{mg}$ orally as an anti-inflammatory and $500 \mathrm{mg}$ of aspirin for 6 days as an anticoagulant. Three of the patients had to be nebulized with ipratropium bromide/ salbutamol, 3 times a day for 5 days, as they had difficulty breathing. Two of the patients had to be given oxygen (4 Lpm) as they had an oxygen saturation below $90 \%$.

After 14 days of treatment, they were tested for $\mathrm{IgG}$ and IgM antibodies to SARS-Cov-2, all were positive for $\mathrm{IgG}$ antibodies.

\section{Discussion}

In this study, 15 patients were treated with amantadine as an antiviral. All patients recovered successfully with amantadine treatment, were not required to go to the hospital, and were not connected to a mechanical ventilator.

Studies have shown that amantadine is effective in decreasing the effects caused by coronavirus [13]. It has been shown that people with Parkinson's disease who have been positive for COVID-19, have not exhibited symptoms $[10,11]$. The effect of amantadine is that it can stop the coronavirus, giving the body the opportunity to generate IgG-type antibodies.

Autopsy reports have demonstrated diffuse alveolar damage, edema, hyaline membrane formation, accumulation of megakaryocytes, platelets and neutrophils in the alveolar capillaries, generating pulmonary thrombotic microangiopathy that often results in fibrin thrombi in the alveolar capillaries and small pulmonary arteries [15].

For this reason, azithromycin was added to amantadine treatment as an antibiotic capable of reducing the exaggerated production of macrophages and pro-inflammatory cytosines [16] as well as preventing bacterial aggregation generated in respiratory processes [17].

Also, as part of the treatment, aspirin was used as an anticoagulant to reduce the risk of venous thromboembolism [18]. Celecoxib was used in most patients as a protective measure for the vascular endothelium [19].

Three patients received ipratropium bromide combined with salbutamol, because they presented respiratory distress [20]. None of the patients were administered dexamethasone [21], because steroids inhibit the immune response and the effect we expect with amantadine was that the virus would stop and the body would develop IgGtype antibodies against the coronavirus.

\section{Conclusion}

It is important to point out that amantadine is an effective antiviral that can stop the symptoms of the coronavirus and gives the body the opportunity to defend itself by generating protective antibodies. Family members reported that they did not have the symptoms generated by the coronavirus.

Amantadine can be used as a viable and cost-effective alternative for treating people with severe acute respiratory syndrome (SARS-Cov-2) on an ambulatory basis, while the vaccine is not available.

Author contributions GEA-A: Project design and article writing. JDA$\mathrm{M}$ : consultation and follow-up of patients. RAL patient monitoring. MEH-A: discussion of results. DH-Co: data collection. FR-D: data collection.

Funding No funding.

\section{Compliance with ethical standards}

Conflict of interest The authors declare that they have no competing interests. 


\section{References}

1. Roser M, Ritchie H, Ortiz-Ospina E, Hasell J. Coronavirus disease (COVID-19)—statistics and research. 2020. https://ourwo rldindata.org/coronavirus

2. Gautret P, Lagier JC, Parola P, Hoang VT, Meddeb L, Maihe $\mathrm{M}$ et al. Hydroxychloroquine and azithromycin as a treatment of COVID-19: results of an open-label non-randomized clinical trial. Int J Antimicrob Agents. 2020;56(1):105949.

3. Schmith VD, Zhou JJ, Lohmer LRL. The approved dose of ivermectin alone is not the ideal dose for the treatment of COVID-19. Clin Pharmacol Ther. 2020;108(4):762-5.

4. FDA US. Coronavirus (COVID-19) update: FDA issues emergency use authorization for potential COVID-19 treatment. 2020. https://www.fda.gov/news-events/press-announcements/coron avirus-covid-19-update-fda-issues-emergency-use-authorizat ion-potential-covid-19-treatment.

5. Nisar T, Sutherland-Foggio H, Husar W. Antiviral amantadine. Lancet Neurol. 2019;18(12):1080.

6. McKimm-Breschkin JL, Fry AM. Meeting report: 4th ISIRV antiviral group conference: novel antiviral therapies for influenza and other respiratory viruses. Antivir Res. 2016;129:21-38.

7. Araújo R, Aranda-Martínez JD, Aranda-Abreu GE. Amantadine treatment for people with COVID-19. Arch Med Res. 2020. https ://doi.org/10.1016/j.arcmed.2020.06.009.

8. Duwe S. Influenza viruses - antiviral therapy and resistance. GMS Infect Dis. 2017;5:Doc04.

9. Rascol O, Negre-Pages L, Damier P, Delval A, Derkinderen P, Destée A, et al. Utilization patterns of amantadine in Parkinson's disease patients enrolled in the French COPARK study. Drugs Aging. 2020;37(3):215-23.

10. Rejdak K, Grieb P. Adamantanes might be protective from COVID-19 in patients with neurological diseases: multiple sclerosis, parkinsonism and cognitive impairment. Mult Scler Relat Disord. 2020;42:102163.

11. Cortés BA. Does amantadine have a protective effect against COVID-19. Neurol Neurochir Pol. 2020;54(3):284-5.

12. Aranda Abreu GE, Hernández Aguilar ME, Herrera Covarrubias D, Rojas DF. Amantadine as a drug to mitigate the effects of COVID-19. Med Hypotheses. 2020;140:109755.
13. Aranda-Abreu GE, Aranda-Martínez JD, Araújo R. Use of amantadine in a patient with SARS-CoV-2. J Med Virol. 2020. https:// doi.org/10.1002/jmv.26179.

14. von Elm E, Altman DG, Egger M, Pocock SJ, Gotzsche PC, Vandenbroucke JP, et al. The Strengthening the Reporting of Observational Studies in Epidemiology (STROBE) statement: guidelines for reporting observational studies. J Clin Epidemiol. 2008;61(4):344-9.

15. Barth R, Maximillian Buja L, Parwani A. The spectrum of pathological findings in coronavirus disease (COVID-19) and the pathogenesis of SARS-CoV-2. Diagn Pathol. 2020;15(1):85.

16. Meyer M, Huaux F, Gavilanes X, Van den Brule S, Lebecque $\mathrm{P}$, Lo Re S, et al. Azithromycin reduces exaggerated cytokine production by M1 alveolar macrophages in cystic fibrosis. Am J Respir Cell Mol Biol. 2009;41(5):590-602.

17. Welte T. Azithromycin: the holy grail to prevent exacerbations in chronic respiratory disease. Am J Respir Crit Care Med. 2019;200(3):269-70.

18. Marik PE, Cavallazzi R. Extended anticoagulant and aspirin treatment for the secondary prevention of thromboembolic disease: a systematic review and meta-analysis. PLoS ONE. 2015;10(11):e0143252.

19. Al-Rashed F, Calay D, Lang M, Thornton CC, Bauer A, Kiprianos A, et al. Celecoxib exerts protective effects in the vascular endothelium via COX-2-independent activation of AMPK-CREBNrf2 signalling. Sci Rep. 2018;8(1):6271.

20. Wolkove N, Kamel H, Rotaple M, Baltzan MA. Use of a mucus clearance device enhances the bronchodilator response in patients with stable COPD. Chest. 2002;121(3):702-7.

21. Coutinho AE, Chapman KE. The anti-inflammatory and immunosuppressive effects of glucocorticoids, recent developments and mechanistic insights. Mol Cell Endocrinol. 2011;335(1):2-13.

Publisher's Note Springer Nature remains neutral with regard to jurisdictional claims in published maps and institutional affiliations. 